\title{
Cardiac catheterization within 1 to 3 days of proximal aortic surgery is not associated with increased postoperative acute kidney injury
}

\author{
Nicholas D. Andersen, MD, ${ }^{a}$ Judson B. Williams, MD, ${ }^{\text {a,b }}$ Emil L. Fosbol, MD, PhD, ${ }^{\mathrm{b}}$ Asad A. Shah, MD, ${ }^{\mathrm{a}}$ \\ Syamal D. Bhattacharya, MD, ${ }^{a}$ Rajendra H. Mehta, MD, MS, ${ }^{b}$ and G. Chad Hughes, MD ${ }^{a}$
}

\begin{abstract}
Objective: Cardiac catheterization shortly before coronary artery bypass grafting or valve surgery has been associated with increased postoperative acute kidney injury. The relationship between catheterization timing and acute kidney injury after proximal aortic surgery remains unknown.
\end{abstract}

\begin{abstract}
Methods: Between August 2005 and February 2011, a total of 285 consecutive patients underwent cardiac catheterization before elective proximal aortic surgery with cardiopulmonary bypass at a single institution. The association between timing of catheterization and postoperative acute kidney injury (defined as postoperative increase in serum creatinine $\geq 50 \%$ of baseline) was assessed using logistic regression analysis.
\end{abstract}

\begin{abstract}
Results: Of 285 patients, $152(53 \%)$ underwent catheterization on preoperative days 1 to 3 and $133(47 \%)$ underwent catheterization on preoperative day 4 or before. Acute kidney injury occurred in $88(31 \%)$ patients, 3 $(1.1 \%)$ requiring dialysis. Acute kidney injury occurred in $37(24 \%)$ patients catheterized on preoperative days 1 to 3 , and 51 (38\%) patients catheterized on preoperative day 4 or before. Catheterization on preoperative days 1 to 3 was not associated with an increased risk of acute kidney injury relative to catheterization on preoperative day 4 or before (unadjusted odds ratio, $0.52 ; 95 \%$ confidence interval, $0.31-0.86 ; P=.01$; adjusted odds ratio, $0.35 ; 95 \%$ confidence interval, $0.17-0.73 ; P=.005)$.
\end{abstract}

Conclusions: Cardiac catheterization within 1 to 3 days of elective proximal aortic surgery appears safe and should be considered acceptable practice for patients at low risk of acute kidney injury. (J Thorac Cardiovasc Surg 2012;143:1404-10)

Acute kidney injury (AKI) occurs in $18 \%$ to $48 \%$ of patients after thoracic aortic surgery and is associated with increased hospital costs, lengths of stay, and mortality. $^{1-4}$ Cardiac catheterization is routinely performed before elective surgery of the proximal thoracic aorta to define aortic root and coronary anatomy and screen for coronary artery disease requiring concomitant intervention. ${ }^{5}$ A number of recent observational studies have suggested cardiac catheterization within 0 to 5 days of coronary artery bypass grafting (CABG) or valve surgery leads to an increased risk of postoperative AKI, presumably from the combined insult of contrast-induced acute kidney injury (CI-AKI) and the physiologic stresses of cardiac surgery and cardiopulmonary bypass $(\mathrm{CPB}) .^{6-11}$

\footnotetext{
From the Division of Thoracic and Cardiovascular Surgery, ${ }^{a}$ Duke Clinical Research Institute, ${ }^{\mathrm{b}}$ Duke University Medical Center, Durham, NC.

Funded by the Thoracic Surgery Foundation for Research and Education (Beverly, Mass) Research Fellowship (to N.D.A.), National Institutes of Health (Bethesda, Md) grants T32-HL069749 (to J.B.W.), U01-HL088953 (to J.B.W.), and T32CA093245 (to S.D.B.).

Received for publication Sept 2, 2011; revisions received Dec 17, 2011; accepted for publication Jan 24, 2012; available ahead of print Feb 20, 2012.

Address for reprints: G. Chad Hughes, MD, Director, Aortic Surgery Program, Duke University Medical Center, Division of Thoracic and Cardiovascular Surgery, Box 3051, Durham, NC 27710 (E-mail: gchad.hughes@ duke.edu).

$0022-5223 / \$ 36.00$

Copyright (C) 2012 by The American Association for Thoracic Surgery doi: $10.1016 /$ j.jtcvs.2012.01.069
}

Patients undergoing proximal thoracic aortic procedures represent a distinct cohort of the cardiac surgery population. A high proportion of individuals harbor aortic disease of congenital or hereditary origin, and the majority of operations require deep hypothermic circulatory arrest (DHCA). ${ }^{12}$ Further, preoperative cardiac catheterization often includes aortic angiogram in addition to coronary and left ventricular angiography, resulting in higher average contrast volumes than in patients undergoing cardiac catheterization for $\mathrm{CABG}$ or valve surgery. We therefore sought to assess the relationship between the timing of preoperative cardiac catheterization before elective proximal thoracic aortic surgery and the incidence of postoperative AKI in this disparate patient group theoretically at a greater risk of AKI.

\section{METHODS}

\section{Patient Population and Data Collection}

The Duke Thoracic Aortic Surgery Database is a prospectively maintained electronic clinical registry of all patients who have undergone a thoracic aortic procedure at Duke University Medical Center (Durham, NC) since July 2005. Preoperative and intraoperative variables are recorded at the time of surgery and postoperative events are ascertained 30 days after hospital discharge. Data accuracy and adjudication of postoperative events are verified by at least 2 independent surgeon investigators. Long-term follow-up and life status are assessed from the medical record and the Social Security Death Index.

This study was approved by the Institutional Review Board of Duke University and the need for individual patient consent was waived. A query 


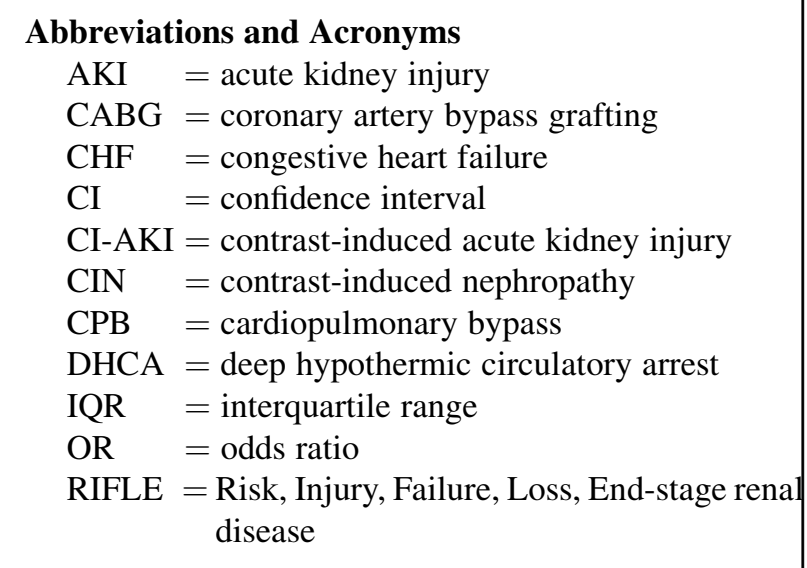

of the Duke Thoracic Aortic Surgery Database identified 293 consecutive elective proximal thoracic aortic procedures performed between August 2005 and February 2011 at our institution via median sternotomy with the use of CPB and without the use of intraoperative contrast agents. Individual medical records were reviewed retrospectively to obtain detailed catheterization and preadmission data. Two patients receiving preoperative dialysis and 1 patient who died on postoperative day 0 were excluded from the study. Of the remaining 290 patients, 285 (98\%) underwent preoperative cardiac catheterization and form the basis of this report.

\section{Catheterization and Preadmission Protocol}

Preoperative cardiac catheterization was performed at our institution in $216(76 \%)$ patients and at an outside institution in the remainder. If not already performed before referral, cardiac catheterization was arranged by the cardiac surgeon and timing determined by patient convenience as well as comorbidities. Catheterization was not performed on the same day as the operation. The routine catheterization protocol at our institution includes discontinuation of nephrotoxic drugs 48 hours before the procedure, prehydration with intravenous normal saline, use of $\mathrm{N}$-acetyl cysteine for patients with serum creatinine of $1.5 \mathrm{mg} / \mathrm{dL}$ or more, use of low volumes of low-osmolar (iopamidol) or iso-osmolar (iodixanol) nonionic contrast agents, and posthydration with $1 \mathrm{~L}$ of intravenous normal saline in patients not at risk for volume overload. Patients catheterized the day before the operation are frequently admitted for overnight hydration with intravenous saline, $0.9 \% 1 \mathrm{~mL} \cdot \mathrm{kg}^{-1} \cdot \mathrm{h}^{-1}$, up until the time of surgery. Irrespective of catheterization, patients with congestive heart failure (CHF) are routinely preadmitted for volume optimization with intravenous diuretics, and patients with chronic kidney disease are routinely preadmitted for intravenous hydration.

\section{Clinical Definitions}

The most recent creatinine level recorded before surgery was defined as the baseline. Postoperative creatinine levels were measured at least once daily until hospital discharge. Postoperative AKI was defined by achieving any Acute Dialysis Quality Initiative Workgroup RIFLE (Risk, Injury, Failure, Loss, End-stage renal disease) criteria for acute renal failure (earliest " $\mathrm{R}=$ risk" stage: plasma creatinine increase $\geq 1.5 \times$ baseline) based on differences between the baseline and peak postoperative serum creatinine levels. ${ }^{13}$ Rates of acute renal failure by The Society of Thoracic Surgeons criteria (postoperative serum creatinine $\geq 2 \times$ baseline and $>2.0 \mathrm{mg} / \mathrm{dL}$ ) and new-onset dialysis were also recorded. Patient characteristics and comorbidities were defined using The Society of Thoracic Surgeons definitions. Estimated glomerular filtration rate was calculated using the Modification of Diet in Renal Disease formula. ${ }^{14}$ The contrast-induced nephropathy (CIN) risk score was calculated as described by Mehran and associates (Figure 1). ${ }^{15}$

\section{Statistical Analysis}

Preoperative and intraoperative variables were compared between patients undergoing cardiac catheterization greater than (long interval) or less than (short interval) 72 hours before surgery. The cutoff between short- and long-interval catheterization was chosen a priori to capture the postcatheterization period at highest risk of CI-AKI and the preoperative catheterization period associated with postoperative $\mathrm{AKI}$ in most prior studies. ${ }^{6-11,16}$ Given that CI-AKI occurs within 3 days of angiography, ${ }^{17}$ for patients in the short-interval catheterization group the period of CI-AKI after catheterization should overlap with surgery and potentially lead to an increased incidence of postoperative AKI. For patients in the long-interval catheterization group, CI-AKI should theoretically have resolved before surgery and should not contribute to the incidence of postoperative AKI. However, CI-AKI incidence and resolution could not be directly assessed given the lack of serial creatinine recordings between catheterization and surgery for most patients. Distribution of baseline characteristics was compared using the parametric $\chi^{2}$ test and the Kruskal-Wallis test for normally and nonnormally distributed continuous variables, respectively. Binary variables were compared using the Cochran-Armitage trend test.

The association between timing of catheterization and AKI was assessed using a logistic regression model. In multivariable models, estimates were adjusted for the following risk factors for AKI after cardiac or thoracic aortic surgery: age, sex, body mass index, baseline estimated glomerular filtration rate, hypertension, diabetes mellitus, CHF, CPB duration, use of DHCA, ejection fraction, preoperative hemoglobin, and aprotinin exposure. $^{1-3,7,18}$ Concomitant CABG was further included in the model as a surrogate for coronary artery disease and because patients undergoing CABG surgery have been shown to be at higher risk of AKI with shortinterval catheterization. ${ }^{6-9,11}$ Intravenous hydration within 24 hours of operation was included to adjust for this potential renoprotective adjunct ${ }^{19}$ that was highly associated with short-interval catheterization. The volume of contrast administered during catheterization was not included given that this information was unavailable for patients catheterized at outside institutions. Underlying assumptions of the logistic regression model were tested and valid unless otherwise indicated (including important interactions: age, sex, and concomitant $\mathrm{CABG}$ ). Calculations were performed using SAS 9.2 (SAS Institute, Inc, Cary, NC).

\section{RESULTS}

Patient clinical and procedural characteristics are reported in Table 1 and Table 2. One hundred sixty-one $(56 \%)$ patients required an operation for a congenital or hereditary condition (bicuspid aortic valve syndrome, 138 [48\%]; hereditary connective tissue disease, 22 [8\%]; coarctation, $1[0.4 \%])$, whereas $124(44 \%)$ patients had acquired or degenerative disease. In addition to proximal aortic operation, $53(19 \%)$ patients underwent concomitant $\mathrm{CABG}$, and $31(11 \%)$ patients underwent 34 non-CABG concomitant procedures (12 arch vessel bypasses, 7 maze procedures, 6 atrial septal defect repairs, 3 mitral valve replacements, 3 tricuspid valve repairs, 1 mitral valve repair, 1 ascending-descending aortic bypass, and 1 video-assisted thoracoscopic wedge resection).

Of the 285 patients included in the analysis, $152(53 \%)$ underwent catheterization on preoperative days 1 to 3 (127 [45\%] on preoperative day $1,7[2 \%]$ on preoperative day 2 , and $18[6 \%]$ on preoperative day 3$)$ and $133(47 \%)$ 


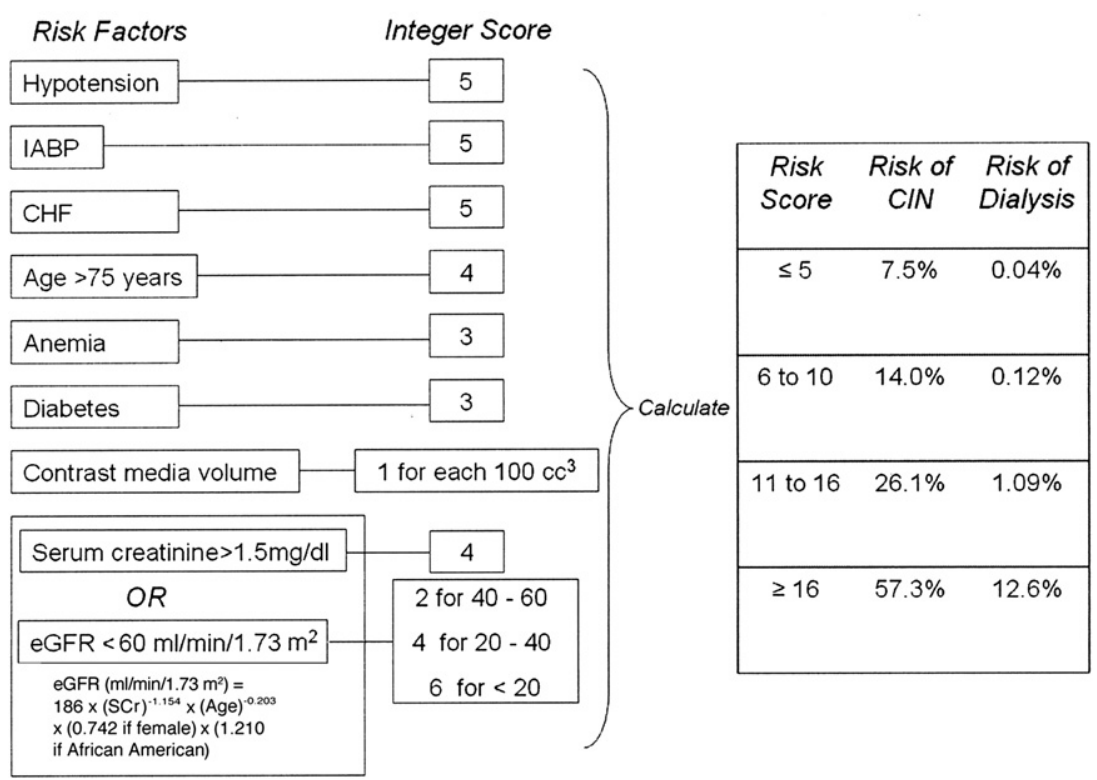

FIGURE 1. Scheme to define contrast-induced nephropathy (CIN) risk score. Anemia, Baseline hematocrit value $<39 \%$ for men and $<36 \%$ for women; $C H F$, congestive heart failure class III/IV by New York Heart Association classification and/or history of pulmonary edema; $e G F R$, estimated glomerular filtration rate; hypotension, systolic blood pressure $<80 \mathrm{~mm} \mathrm{Hg}$ for at least 1 hour requiring inotropic support with medications or intra-aortic balloon pump $(I A B P)$ within 24 hours periprocedurally. (Reprinted with permission. ${ }^{15}$ )

underwent catheterization on preoperative day 4 or before (median preoperative day, 30; interquartile range [IQR], 15-54). Data on the volume of contrast administered during catheterization were available for $152(100 \%)$ of $152 \mathrm{pa}-$ tients having short-interval catheterization and $63(47 \%)$ of 133 having long-interval catheterization. The median overall contrast dose was $1.55 \mathrm{~mL} / \mathrm{kg}$ (IQR, 1.09-2.13 $\mathrm{mL} / \mathrm{kg}$ ). Patients having short-interval catheterization received lower contrast volumes than those having longinterval catheterization $(1.47 \mathrm{~mL} / \mathrm{kg}$ [IQR, $1.06-2.03$ $\mathrm{mL} / \mathrm{kg}$ ] vs $1.81 \mathrm{~mL} / \mathrm{kg}$ [IQR, $1.22-2.38 \mathrm{~mL} / \mathrm{kg}$ ]; $P=.01$ ).

Unadjusted measures of AKI and mortality are reported in Table 3. AKI by any RIFLE criteria occurred in 88 $(31 \%)$ individuals. AKI was an early postoperative event and occurred on postoperative days 0 to 3 in $79(90 \%)$ patients, on postoperative days 4 to 7 in $8(9 \%)$ patients, and after postoperative day 7 in $1(1 \%)$ patient. New-onset dialysis occurred in $3(1.1 \%)$ patients who were catheterized on preoperative days 1,6 , and 14 , respectively. Thirty-day/inhospital mortality occurred in $5(1.8 \%)$ patients, of whom 3 $(60 \%)$ experienced AKI.

The incidence of AKI was significantly lower in patients undergoing catheterization on preoperative days 1 to $3 \mathrm{com}$ pared with patients catheterized on preoperative day 4 or before $(24 \%$ vs $38 \%)$. After adjusting for known risk factors for AKI and other pertinent imbalances between the patient groups, short-interval catheterization was associated with a lower risk of postoperative AKI when compared with long-interval catheterization (odds ratio [OR], 0.35;
$95 \%$ confidence interval $[\mathrm{CI}], 0.17-0.73 ; P=.005$; Table 4). A subgroup analysis was performed by dividing patients into 2 risk groups on the basis of median CIN risk score. No association between catheterization timing and AKI was identified for low-risk patients (CIN risk score, 0-2; OR, $0.44 ; 95 \%$ CI, $0.15-1.28 ; P=.10$ ). However, shortinterval catheterization remained associated with a lower risk of postoperative AKI in the higher-risk subgroup (CIN risk score $\geq 3$; OR, 0.31; 95\% CI, 0.10-0.93; $P=0.04)$.

\section{DISCUSSION}

This observational single-institution study of a large contemporary cohort of patients undergoing complex elective proximal thoracic aortic operations found cardiac catheterization within 1 to 3 days of surgery was not associated with an increased risk of postoperative AKI.

Although the incidence of postoperative AKI (31\%) in our study appears higher than in the comparable studies of CABG or valve surgery, ${ }^{6-11}$ it is similar to the AKI rates reported in prior studies of complex thoracic aortic operations. ${ }^{1-4}$ However, the incidence of dialysis $(1.1 \%)$ in our study remained low, consistent with the expected rates of dialysis in low-risk patients undergoing elective operation. Although AKI is an established risk factor for mortality after cardiac and thoracic aortic operations, ${ }^{1-4,11}$ our study is underpowered to redemonstrate this association in multivariable models given that only $5(1.8 \%)$ postoperative deaths occurred. Nonetheless, it is assumed 
TABLE 1. Clinical features

\begin{tabular}{|c|c|c|c|c|}
\hline Variable & Overall $(\mathbf{n}=\mathbf{2 8 5})$ & Cath on preop days 1-3 $(n=152)$ & Cath on preop day $4+(n=133)$ & $P$ value \\
\hline \multicolumn{5}{|l|}{ Patient characteristics } \\
\hline Age (y) & $59(47-68)$ & $57(46-66)$ & $60(49-69)$ & .04 \\
\hline Female sex & $84(29 \%)$ & $44(29 \%)$ & $40(30 \%)$ & .84 \\
\hline White race & $233(82 \%)$ & $128(84 \%)$ & $105(79 \%)$ & .54 \\
\hline Body mass index $\left(\mathrm{kg} / \mathrm{m}^{2}\right)$ & $27(24-32)$ & $26(24-31)$ & $28(24-33)$ & .04 \\
\hline \multicolumn{5}{|l|}{ Patient comorbidities } \\
\hline Hypertension & $215(75 \%)$ & $115(76 \%)$ & $100(75 \%)$ & .93 \\
\hline Diabetes & $21(7 \%)$ & $9(6 \%)$ & $12(9 \%)$ & .32 \\
\hline Smoker & $110(39 \%)$ & $59(39 \%)$ & $51(38 \%)$ & .94 \\
\hline Congestive heart failure & $26(9 \%)$ & $8(5 \%)$ & $18(14 \%)$ & .02 \\
\hline Prior myocardial infarction & $15(5 \%)$ & $5(3 \%)$ & $10(8 \%)$ & .11 \\
\hline Prior stroke & $19(7 \%)$ & $10(7 \%)$ & $9(7 \%)$ & .95 \\
\hline Chronic obstructive pulmonary disease & $36(13 \%)$ & $19(13 \%)$ & $17(13 \%)$ & .94 \\
\hline Peripheral vascular disease & $9(3 \%)$ & $4(3 \%)$ & $5(4 \%)$ & .59 \\
\hline Gastroesophageal reflux disease & $68(24 \%)$ & $36(24 \%)$ & $32(24 \%)$ & .94 \\
\hline Chronic alcohol use & $19(7 \%)$ & $9(6 \%)$ & $10(8 \%)$ & .59 \\
\hline Prior aortic surgery & $69(24 \%)$ & $49(32 \%)$ & $20(15 \%)$ & .001 \\
\hline Contrast-induced nephropathy risk score & $2(1-5)$ & $2(1-4.5)$ & $3(0-6)$ & .97 \\
\hline \multicolumn{5}{|l|}{ Medications } \\
\hline Long-term steroid use & $4(1 \%)$ & $2(1 \%)$ & $2(2 \%)$ & .89 \\
\hline Preop diuretic & $75(26 \%)$ & $35(23 \%)$ & $40(30 \%)$ & .18 \\
\hline Preop ACEi/ARB & $110(39 \%)$ & $61(40 \%)$ & $49(37 \%)$ & .57 \\
\hline \multicolumn{5}{|l|}{ Baseline values } \\
\hline Ejection fraction & $55(55-55)$ & $55(55-55)$ & $55(55-55)$ & .11 \\
\hline Hemoglobin (g/dL) & $13.9(12.9-15.0)$ & $13.9(13.0-14.9)$ & $14.1(12.8-15.1)$ & .70 \\
\hline Creatinine (mg/dL) & $1.0(0.8-1.1)$ & $0.9(0.8-1.1)$ & $1.0(0.9-1.1)$ & .19 \\
\hline$>1.5$ & $12(4 \%)$ & $5(3 \%)$ & $7(5 \%)$ & .41 \\
\hline eGFR $\left(\mathrm{mL} \cdot \min ^{-1} \cdot 1.73 \mathrm{~m}^{-2}\right)$ & $80(69-94)$ & $80(71-96)$ & $78(68-92)$ & .11 \\
\hline$>60$ & $248(87 \%)$ & $136(89 \%)$ & $112(84 \%)$ & .19 \\
\hline $40-60$ & $34(12 \%)$ & $14(9 \%)$ & $20(15 \%)$ & .13 \\
\hline $20-40$ & $3(1 \%)$ & $2(1 \%)$ & $1(0.8 \%)$ & .64 \\
\hline
\end{tabular}

Values expressed as median (interquartile range) or number (percent). $A C E i$, Angiotensin-converting enzyme inhibitor; $A R B$, angiotensin receptor blocker; $e G F R$, estimated glomerular filtration rate.

from prior studies that postoperative AKI leads to worse patient outcomes, and aggressive efforts should be taken to minimize AKI in the perioperative period.

There were many differences between the short- and long-interval catheterization groups that we attempted to adjust for in multivariable models. Risk factors for AKI with unequal distribution included age (57 vs 60 years), body mass index (26 vs $\left.28 \mathrm{~kg} / \mathrm{m}^{2}\right)$, and CHF (5\% vs $14 \%$ ). A higher proportion of patients with a history of aortic surgery ( $32 \%$ vs $15 \%)$, hereditary connective tissue disease $(11 \%$ vs $3 \%)$, and undergoing redo sternotomy $(30 \%$ vs $17 \%$ ) underwent short-interval catheterization, possibly as a result of younger patient age secondary to congenital or hereditary aortic disease. These differences were likely partially controlled for by adjusting for age and CPB time. A higher proportion of patients undergoing concomitant CABG $(26 \%$ vs $13 \%)$ underwent long-interval catheterization, possibly as a result of comorbidities or due to referral for aortic surgery only after the identification of thoracic aortic disease on catheterization for coronary artery disease.
As a result of our catheterization protocols, a greater proportion of patients undergoing short-interval catheterization were preadmitted to the hospital before surgery $(63 \%$ vs $43 \%$ ) and received intravenous hydration within 24 hours of the operation ( $75 \%$ vs $14 \%$ ). In contrast, a greater proportion of patients undergoing long-interval catheterization received intravenous diuretics before surgery ( $14 \%$ vs $8 \%)$ given that there were more patients with $\mathrm{CHF}$ in this group $(14 \%$ vs $5 \%)$. Concomitant CABG, preoperative hydration, and $\mathrm{CHF}$ were all included in the multivariable models. Last, patients having short-interval catheterization received lower volumes of contrast during catheterization $(1.47 \mathrm{vs} 1.81 \mathrm{~mL} / \mathrm{kg})$, likely as a result of our institutional protocols aimed at minimizing contrast exposure for patients undergoing catheterization shortly before surgery. We were unable to adjust for the effect of contrast volume on postoperative AKI given that this information was missing for $53 \%$ of patients having long-interval catheterization. However, exposure to greater contrast volumes in the long-interval group would not be predicted to increase the 
TABLE 2. Procedural characteristics

\begin{tabular}{|c|c|c|c|c|}
\hline Variable & Overall $(n=285)$ & Cath on preop days 1-3 $(n=152)$ & Cath on preop day $4+(n=133)$ & $P$ value \\
\hline ASA class & & & & .44 \\
\hline 2 & $5(2 \%)$ & $1(1 \%)$ & $4(3 \%)$ & \\
\hline 3 & $224(79 \%)$ & $120(79 \%)$ & $104(78 \%)$ & \\
\hline 4 & $56(20 \%)$ & $31(20 \%)$ & $25(19 \%)$ & \\
\hline Hospital preadmission & $153(54 \%)$ & $96(63 \%)$ & $57(43 \%)$ & .006 \\
\hline IV hydration w/in $24 \mathrm{~h}$ of operation & $133(47 \%)$ & $114(75 \%)$ & $19(14 \%)$ & $<.001$ \\
\hline IV diuretic w/in $24 \mathrm{~h}$ of operation & $31(11 \%)$ & $12(8 \%)$ & $19(14 \%)$ & .08 \\
\hline \multicolumn{5}{|l|}{ Etiology } \\
\hline Bicuspid aortic valve syndrome & $138(48 \%)$ & $70(46 \%)$ & $68(51 \%)$ & .39 \\
\hline Degenerative atherosclerosis & $94(33 \%)$ & $46(30 \%)$ & $48(36 \%)$ & .28 \\
\hline Hereditary connective tissue disease & $22(8 \%)$ & $18(12 \%)$ & $4(3 \%)$ & .005 \\
\hline Idiopathic & $17(6 \%)$ & $10(7 \%)$ & $7(5 \%)$ & .64 \\
\hline Vasculitis & $11(4 \%)$ & $7(5 \%)$ & $4(3 \%)$ & .48 \\
\hline Pseudoaneurysm & $1(0.4 \%)$ & 0 & $1(0.8 \%)$ & .28 \\
\hline Endocarditis & $1(0.4 \%)$ & 0 & $1(0.8 \%)$ & .28 \\
\hline Coarctation & $1(0.4 \%)$ & $1(0.6 \%)$ & 0 & .35 \\
\hline Primary procedure & & & & .36 \\
\hline Root or ascending aorta replacement only & $57(20 \%)$ & $30(20 \%)$ & $27(20 \%)$ & \\
\hline Root/ascending + hemiarch & $203(71 \%)$ & $105(69 \%)$ & $98(74 \%)$ & \\
\hline Root/ascending + total arch & $25(9 \%)$ & $17(11 \%)$ & $8(6 \%)$ & \\
\hline Concomitant CABG & $53(19 \%)$ & $19(13 \%)$ & $34(26 \%)$ & .005 \\
\hline Other concomitant procedure & $31(11 \%)$ & $12(8 \%)$ & $19(14 \%)$ & .08 \\
\hline \multicolumn{5}{|l|}{ Operative characteristics } \\
\hline Maximum aortic diameter $(\mathrm{cm})$ & $5.5(5.1-5.9)$ & $5.5(5.2-5.8)$ & $5.5(5.0-6.0)$ & .76 \\
\hline Redo sternotomy & $67(24 \%)$ & $45(30 \%)$ & $22(17 \%)$ & .01 \\
\hline Crossclamp time (min) & $143(117-168)$ & $140(109-169)$ & $144(121-168)$ & .28 \\
\hline $\mathrm{CPB}$ time (min) & $209(183-240)$ & $209(183-235)$ & $209(184-244)$ & .43 \\
\hline DHCA & $229(80 \%)$ & $123(81 \%)$ & $106(80 \%)$ & .80 \\
\hline Cerebral DHCA time (min) & $17(14-22)$ & $17(14-22)$ & $17(15-22)$ & .37 \\
\hline Systemic DHCA time (min) & $17(14-23)$ & $17(14-23)$ & $17(15-22)$ & .69 \\
\hline Aprotinin use & $63(22 \%)$ & $31(20 \%)$ & $32(24 \%)$ & .46 \\
\hline
\end{tabular}

Values expressed as median (interquartile range) or number (percent). $A S A$, American Society of Anesthesiologists; $I V$, intravenous; $C A B G$, coronary artery bypass grafting; $C P B$, cardiopulmonary bypass; $D H C A$, deep hypothermic circulatory arrest.

rate of AKI given that kidney recovery presumably occurred in the interval between catheterization and surgery.

Six retrospective studies assessing the relationship between the timing of preoperative cardiac catheterization and the incidence of AKI after CABG or valve surgery reported an increased risk of AKI with short-interval catheterization. In 3 studies, this risk was restricted to patients undergoing catheterization within 24 hours of surgery. ${ }^{7,8,10}$ The remaining studies reported that catheterization within 5 days of surgery ${ }^{6,11}$ or during the same admission as surgery ${ }^{9}$ were associated with increased postoperative AKI. As a result, the various study authors have proposed that surgery should be delayed for as long as 7 days after catheterization or until after hospital discharge and readmission.

The explanation for our disparate study findings is likely related to the unique features of patients requiring surgery for proximal thoracic aortic disease, the inclusion of a purely elective patient population, and the absence of patients undergoing same-day catheterization. Many patients requiring proximal thoracic aortic surgery have aortopathy of congenital or hereditary disease and therefore require surgery at a younger age and harbor few comorbidities. The median age of patients in our study was nearly a decade younger than that of patients in the comparable studies of

TABLE 3. Unadjusted measures of AKI and mortality

\begin{tabular}{|c|c|c|c|c|}
\hline Criteria & Overall $(n=285)$ & Cath on preop days 1-3 $(n=152)$ & Cath on preop day $4+(n=133)$ & $P$ value \\
\hline AKI (all RIFLE stages) & $88(31 \%)$ & $37(24 \%)$ & $51(38 \%)$ & .01 \\
\hline STS acute renal failure & $18(6.3 \%)$ & $6(3.9 \%)$ & $12(9.0 \%)$ & .09 \\
\hline New-onset dialysis & $3(1.1 \%)$ & $1(0.7 \%)$ & $2(1.5 \%)$ & .60 \\
\hline Mortality (30-d/in-hospital) & $5(1.8 \%)$ & $3(2.0 \%)$ & $2(1.5 \%)$ & 1 \\
\hline
\end{tabular}

AKI, Acute kidney injury; RIFLE, Risk, Injury, Failure, Loss, End-stage renal disease; STS, The Society of Thoracic Surgeons. 
TABLE 4. Association between day of catheterization and AKI

\begin{tabular}{|c|c|c|c|}
\hline $\begin{array}{c}\text { Cath on preop } \\
\text { days } 1-3 \text { vs. preop day } 4+\end{array}$ & OR & $95 \%$ CI & $\begin{array}{c}P \\
\text { value }\end{array}$ \\
\hline \multicolumn{4}{|l|}{ AKI (all RIFLE stages) } \\
\hline Crude & 0.52 & $0.31-0.86$ & .01 \\
\hline Adjusted & 0.35 & $0.17-0.73$ & .005 \\
\hline \multicolumn{4}{|l|}{ Subgroup analysis (adjusted) } \\
\hline CIN risk score $\leq 2(n=153)$ & 0.44 & $0.15-1.28$ & .10 \\
\hline CIN risk score $>3(n=132)$ & 0.31 & $0.10-0.93$ & .04 \\
\hline
\end{tabular}

CABG and valve surgery (59 years vs 65-68 years), and the median CIN risk score was 2, placing them at the lowest risk for the development of CI-AKI after catheterization. ${ }^{15} \mathrm{Al}-$ though AKI developed after surgery in $31 \%$ of patients, it progressed to kidney failure requiring dialysis in only $1.1 \%$ of them, suggesting that when AKI did occur in this low-risk patient cohort it was typically mild and transient.

Our study was further restricted to patients undergoing elective surgery, and all emergency and urgent procedures were excluded. In all but 1 prior study ${ }^{11}$ patients undergoing urgent procedures were included in the analysis and were more likely to undergo short-interval catheterization. ${ }^{6-10}$ Hence, the association between AKI and short-interval catheterization was likely partially confounded by unstable patients at higher risk of AKI undergoing expedited surgery shortly after catheterization.

Same-day cardiac catheterization and surgery is not offered at our institution for patients undergoing elective proximal thoracic aortic procedures. Ranucci and colleagues ${ }^{7}$ found that the risk of AKI with short-interval catheterization was restricted only to those undergoing surgery on the same day as catheterization. In all other studies, patients having same-day catheterization were not assessed independently and were grouped together with patients catheterized on preoperative day 1 or before. ${ }^{6,8-11}$ Hence, it is possible that the increased risk of AKI associated with short-interval catheterization is due entirely to same-day catheterization, and no such patients were included in our study. Conversely, 2 reports from the Mayo Clinic, conducted among carefully selected patients at low-risk of AKI, found that cardiac catheterization on the same day as elective valve surgery was not associated with postoperative AKI. ${ }^{20,21}$

The largest study to examine the relationship between catheterization timing and postoperative AKI was recently published by Mehta and colleagues ${ }^{11}$ from our institution. Among 2441 patients undergoing elective, isolated CABG, this study found that cardiac catheterization on preoperative days 0 to 5 was associated with increased postoperative AKI. Numerous features of the Mehta study are consistent with our study findings. First, the CABG patients included in the Mehta study were older (median age 66 years) and sicker (33\% with CHF, 33\% with diabetes) than those with proximal aortic disease included in our study and are therefore at greater risk of CI-CKI after catheterization. ${ }^{15}$ Second, the risk of AKI with short-interval catheterization reported by Mehta was quite modest and bordered on nonsignificance, likely owing to the exclusion of all urgent and emergency procedures (OR, 1.11; 95\% CI, $1.02-1.20 ; P=.02$, when day of catheterization was treated as a continuous variable from $\leq 1$ to $\geq 5$ days). Third, sameday catheterizations were included in the Mehta study but were not assessed as an independent group. However, when day of catheterization was treated as a categorical variable, the increased risk of AKI with short-interval catheterization was again restricted only to patients undergoing catheterization on preoperative days 0 to 1 (OR, 1.74; 95\% CI, 1.13-3.68; reference $=$ catheterization on preoperative day 5 or before). Hence, the overriding conclusion from this and other studies may be to avoid short-interval catheterization for $\mathrm{CABG}$ and other high-risk patients, but that short-interval, or even same-day, catheterization may be well tolerated by patients at low risk of CI-AKI who are undergoing elective surgery.

Paradoxically, our study reports a lower risk of postoperative AKI for patients undergoing short-interval catheterization, as opposed to equivalence between the short- and long-interval catheterization groups. On subgroup analysis, the "benefit" of short-interval catheterization was lost in low-risk patients but persisted in higher-risk patients. We can only conclude that this is due to greater preoperative kidney protection and medical optimization in patients undergoing short-interval catheterization and that these benefits are more pronounced in higher-risk patients and outweigh the insult of CI-AKI. Although we adjusted for preoperative hydration in the multivariable models, unmeasured confounders related to hospital preadmission, such as medical optimization, blood pressure and glycemic control, and careful review and discontinuation of nephrotoxic medications, likely account for the reduced risk of AKI in patients subjected to short-interval catheterization. ${ }^{22}$ However, inasmuch as this remains a speculative hypothesis that is untested in our study, we refrain from recommending short-interval catheterization for high-risk patients and instead suggest that long-interval catheterization followed by hospital preadmission for aggressive preoperative renal protection may lead to optimal results.

\section{Clinical Implications}

Given the small number of centers that specialize in thoracic aortic procedures, a patient requiring a thoracic aortic intervention is likely to be referred away from the community hospital to a regional center of excellence. Shortinterval catheterization allows these patients to undergo catheterization and surgery within a single extended episode of care. The benefits of this practice include reduced costs of stay and resource consumption for patients who 
are kept in the hospital between catheterization and surgery and reduced travel and lodging expenses and increased convenience for patients who would otherwise be discharged home between catheterization and surgery. Nonetheless, for patients at high risk of CI-AKI, such as those with baseline renal dysfunction, diabetes, $\mathrm{CHF}$, and age greater than 75 years, long-interval catheterization remains prudent pending further study. In all patients, aggressive CI-AKI prophylaxis and perioperative kidney protection should be used and may negate the harmful effects of short-interval catheterization and reduce postoperative AKI.

\section{Study Limitations}

Our data are observational and therefore inherently limited by a selection bias, inasmuch as patients selected for long-interval catheterization were older and harbored more risk factors for AKI. Although logistic regression was used to adjust for these differences, unmeasured confounders related to patient selection and perioperative renal protection likely account for the higher rate of AKI observed in the long-interval catheterization group, inasmuch as it is unlikely that short-interval catheterization itself is renoprotective. We are also unable to adjust for the volume of contrast administered during catheterization inasmuch as this information was missing from $53 \%$ of the longinterval catheterization patients who were not catheterized at our institution. Intraoperative and postoperative risk factors for AKI, such as hypotension on bypass and vasopressor use, ${ }^{10}$ were also not recorded given that these variables are unable to influence the decision regarding timing of catheterization. Our data are further limited by the constraints of any observational retrospective study and results should be viewed as hypothesis generating and inference regarding causation made with caution.

\section{CONCLUSIONS}

Despite the use of high contrast volumes during catheterization and the complexity of the subsequent operations, cardiac angiography with aggressive CI-AKI prophylaxis can be performed safely within 1 to 3 days of elective proximal thoracic aortic surgery and should be considered acceptable practice for patients at low-risk of AKI.

\section{References}

1. Augoustides JG, Pochettino A, Ochroch EA, Cowie D, Weiner J, Gambone AJ, et al. Renal dysfunction after thoracic aortic surgery requiring deep hypothermic circulatory arrest: definition, incidence, and clinical predictors. J Cardiothorac Vasc Anesth. 2006;20:673-7.

2. Arnaoutakis GJ, Bihorac A, Martin TD, Hess PJ Jr, Klodell CT, Ejaz AA, et al. RIFLE criteria for acute kidney injury in aortic arch surgery. J Thorac Cardiovasc Surg. 2007;134:1554-60; discussion 1560-1.

3. Englberger L, Suri RM, Greason KL, Burkhart HM, Sundt TM 3rd, Daly RC, et al. Deep hypothermic circulatory arrest is not a risk factor for acute kidney injury in thoracic aortic surgery. $J$ Thorac Cardiovasc Surg. 2011;141:552-8.

4. Augoustides JG, Pochettino A, McGarvey ML, Cowie D, Weiner J, Gambone AJ, et al. Clinical predictors for mortality in adults undergoing thoracic aortic surgery requiring deep hypothermic circulatory arrest. Ann Card Anaesth. 2006;9: 114-9.

5. Hiratzka LF, Bakris GL, Beckman JA, Bersin RM, Carr VF, Casey DE Jr, et al. 2010 ACCF/AHA/AATS/ACR/ASA/SCA/SCAI/SIR/STS/SVM guidelines for the diagnosis and management of patients with Thoracic Aortic Disease: a report of the American College of Cardiology Foundation/American Heart Association Task Force on Practice Guidelines, American Association for Thoracic Surgery, American College of Radiology, American Stroke Association, Society of Cardiovascular Anesthesiologists, Society for Cardiovascular Angiography and Interventions, Society of Interventional Radiology, Society of Thoracic Surgeons, and Society for Vascular Medicine. Circulation. 2010;121:e266-369.

6. Del Duca D, Iqbal S, Rahme E, Goldberg P, de Varennes B. Renal failure after cardiac surgery: timing of cardiac catheterization and other perioperative risk factors. Ann Thorac Surg. 2007;84:1264-71.

7. Ranucci M, Ballotta A, Kunkl A, De Benedetti D, Kandil H, Conti D, et al. Influence of the timing of cardiac catheterization and the amount of contrast media on acute renal failure after cardiac surgery. Am J Cardiol. 2008;101:1112-8.

8. Medalion B, Cohen H, Assali A, Vaknin Assa H, Farkash A, Snir E, et al. The effect of cardiac angiography timing, contrast media dose, and preoperative renal function on acute renal failure after coronary artery bypass grafting. $J$ Thorac Cardiovasc Surg. 2010;139:1539-44.

9. Kramer RS, Quinn RD, Groom RC, Braxton JH, Malenka DJ, Kellett MA, et al. Same admission cardiac catheterization and cardiac surgery: is there an increased incidence of acute kidney injury? Ann Thorac Surg. 2010;90:1418-23; discussion 1423-4.

10. Hennessy SA, LaPar DJ, Stukenborg GJ, Stone ML, Mlynarek RA, Kern JA, et al. Cardiac catheterization within 24 hours of valve surgery is significantly associated with acute renal failure. J Thorac Cardiovasc Surg. 2010;140:1011-7.

11. Mehta RH, Honeycutt E, Patel UD, Lopes RD, Williams JB, Shaw LK, et al. Relationship of the time interval between cardiac catheterization and elective coronary artery bypass surgery with postprocedural acute kidney injury. Circulation. 2011;124(11 Suppl):S149-55.

12. Elefteriades JA. Natural history of thoracic aortic aneurysms: indications for surgery, and surgical versus nonsurgical risks. Ann Thorac Surg. 2002;74:S1877-80; discussion S1892-8.

13. Bellomo R, Ronco C, Kellum JA, Mehta RL, Palevsky P. Acute renal failuredefinition, outcome measures, animal models, fluid therapy and information technology needs: the Second International Consensus Conference of the Acute Dialysis Quality Initiative (ADQI) Group. Crit Care. 2004;8:R204-12.

14. Stevens LA, Coresh J, Greene T, Levey AS. Assessing kidney function-measured and estimated glomerular filtration rate. N Engl J Med. 2006;354:2473-83.

15. Mehran R, Aymong ED, Nikolsky E, Lasic Z, Iakovou I, Fahy M, et al. A simple risk score for prediction of contrast-induced nephropathy after percutaneous coronary intervention: development and initial validation. J Am Coll Cardiol. 2004; 44:1393-9.

16. Solomon R, Dauerman HL. Contrast-induced acute kidney injury. Circulation. 2010;122:2451-5.

17. McCullough PA, Stacul F, Becker CR, Adam A, Lameire N, Tumlin JA, et al. Contrast-Induced Nephropathy (CIN) Consensus Working Panel: executive summary. Rev Cardiovasc Med. 2006;7:177-97.

18. Mori Y, Sato N, Kobayashi Y, Ochiai R. Acute kidney injury during aortic arch surgery under deep hypothermic circulatory arrest. J Anesth. 2011;25:799-804.

19. Marathias KP, Vassili M, Robola A, Alivizatos PA, Palatianos GM, Geroulanos S, et al. Preoperative intravenous hydration confers renoprotection in patients with chronic kidney disease undergoing cardiac surgery. Artif Organs. 2006;30:615-21.

20. Brown ML, Holmes DR, Tajik AJ, Sarano ME, Schaff HV. Safety of same-day coronary angiography in patients undergoing elective valvular heart surgery. Mayo Clin Proc. 2007;82:572-4.

21. Greason KL, Englberger L, Suri RM, Park SJ, Rihal CS, Pislaru SV, et al. Safety of same-day coronary angiography in patients undergoing elective aortic valve replacement. Ann Thorac Surg. 2011;91:1791-6.

22. Brown JR, Thompson CA. Contrast-induced acute kidney injury: the at-risk patient and protective measures. Curr Cardiol Rep. 2010;12:440-5. 